\title{
Efeitos da morfina e da metadona associadas à acepromazina em gatas anestesiadas com propofol e halotano e submetidas à ovariossalpingohisterectomia
}

\author{
Effects of morphine and methadone associated with acepromazine in cats anesthetized \\ with propofol and halothane and subjected to ovariossalpingohisterectomia \\ Regiane Manfrinate', Magyda Arabia Araji Dahroug2 ${ }^{2}$ Daniela Cristina Farias ${ }^{3}$, \\ Luiz Paulo dos Santos Vasconcelos ${ }^{3}$, Nicolle Nagle de Sousa Wayhs ${ }^{3}$, Samuel Monzem ${ }^{3}$, \\ Erotides Capistrano da Silva ${ }^{4}$, João Vitor Amorim Galceran ${ }^{3}$, Francineide Bogorni ${ }^{1}$, \\ Roberto Lopes de Souza ${ }^{5}$ \& Luciana Dambrósio Guimarães ${ }^{5}$
}

\section{RESUMO}

Os fármacos utilizados na medicação pré-anestésica são úteis para preparar o paciente para anestesia, causando sedação, analgesia, redução na dose dos anestésicos e menor incidência de efeitos adversos ao paciente. O objetivo deste trabalho foi avaliar os efeitos cardiovasculares e respiratórios, comportamentais, relaxamento muscular, analgesia pós-operatória da morfina ou metadona associadas à acepromazina em gatas anestesiadas com propofol e halotano e submetidas a ovariossalpingohisterectomia. Para isso, foram utilizadas 20 gatas clinicamente sadias, distribuídas em 2 grupos: o grupo I recebeu a associação de acepromazina $(0,05 \mathrm{mg} / \mathrm{kg})$ e metadona $(0,2 \mathrm{mg} / \mathrm{kg})$ e o grupo II recebeu a associação de acepromazina $(0,05 \mathrm{mg} / \mathrm{kg})$ e morfina $(0,2 \mathrm{mg} / \mathrm{kg})$ administradas na mesma seringa por via intramuscular. Foram avaliados frequência cardíaca e respiratória, pressão arterial sistólica, saturação de oxi-hemoglobina, concentração de dióxido de carbono no ar expirado, temperatura retal e tempo de preenchimento capilar, nos períodos pré, trans e pós-operatórios. Pode-se concluir que ambas as associações produziram leve sedação e podem ser utilizadas com segurança em felinos, pois promoveram boa analgesia sem efeitos colaterais.

Descritores: opióides, anestesia, felinos.

\section{ABSTRACT}

The pre-anesthetic medication is useful to prepare the patient for anesthesia, causing sedation, analgesia, reduction of the anesthetic gas doses and a lower incidence of adverse effects to the patient. Such variation of effects is dependent on the combination of drugs used. The aim of this study was to evaluate the cardiovascular and respiratory effects, behavioral, muscle relaxation, postoperative analgesia and influence on the quality and recovery period of morphine or methadone. For that were used 20 clinically healthy cats, divided into 2 groups: group I received the combination of acepromazine $(0.05 \mathrm{mg} / \mathrm{kg})$ and methadone $(0.2 \mathrm{mg} / \mathrm{kg})$ and group II received a combination of acepromazine $(0.05 \mathrm{mg} / \mathrm{kg})$ and morphine $(0.2 \mathrm{mg} / \mathrm{kg})$ administered in the same syringe for intramuscular injection. It was concluded that both associations produced mild sedation and can be used safely in cats, therefore providing good analgesia and muscle relaxation, without side effects.

Keywords: opioids, anesthesia, felines.

${ }^{1}$ Pós-graduação, curso de Especialização Lato Sensu, Faculdade de Medicina Veterinária (FAMEV), Universidade Federal do Mato Grosso (UFMT), Av. Fernando Correa da Costa s/n., Coxipó, CEP 78060-900 Cuiabá, Mato Grosso, MT, Brasil. ²ós-graduação, Mestrado em Ciências Veterinárias, UFMT. ${ }^{3}$ Graduação, Medicina Veterinária (FAMEV), UFMT. ${ }^{4}$ Pós-graduação, Curso de Ciências Veterinárias, Universidade Federal de Viçosa (UFV), Viçosa, MG, Brasil. '5 Departamento de Clínica Médica Veterinária, FAMEV-UFMT. CORRESPONDÊNCIA: R. Manfrinate [manfrinate@yahoo.com.br ; Fax: +55 (65) 3615 8614]. 


\section{INTRODUÇÃO}

Os fármacos pré-anestésicos (MPA) são úteis na preparação do paciente para anestesia, causando sedação, analgesia e menor incidência de efeitos adversos [4].

Os opióides, além de analgésicos, deprimem o sistema nervoso central produzindo sedação e sonolência [11]. São conhecidos diversos tipos de receptor opiáceos: ти 1 e 2, kappa, delta e sigma [16], sendo que os efeitos do ultimo ainda não estão elucidados [4]. O receptor $m u$ é responsável pela analgesia supraespinal, depressão respiratória, euforia e dependência física; os receptores kappa são responsáveis pela analgesia espinal, miose, sedação e disforia e os receptores sigmas são responsáveis por disforia, alucinação e estímulo vasomotor e respiratório [17].

Pertencente à classe dos opióides pode-se citar a metadona, que atua como antagonista de receptores NMDA (N-metil-D aspartato) na medula espinhal, podendo incrementar a analgesia e diminuir a ocorrência de tolerância pelo seu uso contínuo [24]. É um fármaco seguro por via endovenosa ou epidural, com poucos efeitos secundários [18]. Outro opióide importante, a morfina, causa analgesia com certo grau de sedação e alívio da dor, seja no período pré-anestésico ou no transoperatório [6,17]. Pode ocorrer náusea e vômito que estão relacionados à estimulação da zona deflagradora dos quimiorreceptores localizadas nas paredes laterais do terceiro ventrículo [6].

O objetivo deste trabalho foi avaliar os efeitos da morfina ou metadona associados à acepromazina sobre a anestesia com propofol e halotano em gatas submetidas à ovariossalpingohisterectomia.

\section{MATERIAS E MÉTODOS}

Utilizaram-se vinte gatas clinicamente sadias, sem raça definida, adultas, classificadas como ASA I, atendidas no Hospital Veterinário da Universidade Federal do Mato Grosso (UFMT), submetidas a procedimentos de ovariossalpingohisterectomia eletiva. Foi instituído um jejum sólido de 12 horas e hídrico de 4 horas. Estes animais foram divididos em dois grupos denominados GI $\left(0,05 \mathrm{mg} / \mathrm{kg}\right.$ acepromazina ${ }^{1}$ e $0,2 \mathrm{mg} / \mathrm{kg}$ metadona $\left.^{2}\right)$ e GII $(0,05 \mathrm{mg} / \mathrm{kg}$ acepromazina e $0,2 \mathrm{mg} / \mathrm{kg}$ morfina $^{3}$ ) administrados por via intramuscular como medicação pré-anestésica. Decorridos 15 minutos, foi realizada a indução da anestesia através da administração de $5 \mathrm{mg} / \mathrm{kg}$ de propofol $^{4}$ intravenoso. Assim que apresentaram relaxamento da região mandibular e ausência do reflexo laringotraqueal, foi realizada a intubação orotraqueal com sonda apropriada, e a mesma foi conectada ao circuito de anestesia Baraka, por onde os animais receberam halotano ${ }^{5}$ diluído em $100 \mathrm{ml} /$ $\mathrm{kg} / \mathrm{h}$ de oxigênio a $100 \%$ e foram mantidos no $3^{\circ}$ plano de anestesia. Para fluidoterapia os animais receberam solução de Ringer com lactato na velocidade de $10 \mathrm{ml} / \mathrm{kg} / \mathrm{h}$ intravenoso.

Foram avaliadas as seguintes variáveis: frequência e ritmo cardíaco (através de eletrocardiógrafo para computador $^{6}$ ); temperatura retal (TR - com auxilio de termômetro digital); pressão arterial sistólica (PAS com Doppler ultrassônico ${ }^{7}$ ); frequência respiratória (FR - através dos movimentos da caixa torácica); saturação periférica de oxi-hemoglobina $\left(\mathrm{SpO}_{2}\right.$ - através de oximetria de pulso ${ }^{8}$ com o sensor posicionado na língua do animal); concentração de dióxido de carbono no ar expirado $\left(\mathrm{ETCO}_{2}\right.$ - através de capnografia). Essas variáveis foram mensuradas nos seguintes tempos: imediatamente antes da MPA (valores basais); 15 minutos após MPA; aos 10, 20, 30 e 40 minutos após a indução da anestesia; aos 30, 60, 90 e 120 minutos após o término da cirurgia. A saturação da oxi-hemoglobina, concentração de dióxido de carbono expirado e eletrocardiografia foram avaliadas após a indução da anestesia.

Durante todo o período experimental, foi avaliado o comportamento, o relaxamento muscular, as características da recuperação anestésica e o grau de analgesia através de sistemas de escore.

Para avaliação do comportamento de cada animal durante o período pré-operatório (imediatamente antes e 15 minutos após a MPA), foi utilizado um sistema de escore em que: $0=$ representava animais atentos a todos os movimentos da sala e se mantendo em pé; $1=$ animal sonolento e sem coordenação dos membros e $2=$ animal em decúbito esternal e dormindo. Para avaliação do grau de relaxamento muscular no pré e transoperatório: $0=$ tônus do membro e mandíbula normais; $1=$ discreto relaxamento muscular; $2=$ moderado relaxamento muscular e $3=$ intenso relaxamento muscular.

Durante o pós-operatório, os animais foram avaliados a cada 30 minutos, durante duas horas, em relação à capacidade em se manter em decúbito esternal, capacidade de se locomover com ou sem ataxia, incidência de vocalização, vômito, defecação, comportamento de fuga ou agitação, e escore de dor. Para monitorar a analgesia no pós-operatório, foi utilizado 
o sistema de escore de dor 0 a 3, sendo considerado: $0=$ sem dor, sem sinais de desconforto e sem resistência a pressão firme; 1=dor leve, sem sinais de desconforto, mas resiste à pressão firme; $2=$ dor moderada, algum sinal de desconforto que pioram pela pressão firme e, $3=$ dor intensa, óbvios sinais de desconforto persistente que pioram pela pressão firme [9]. Aqueles animais que demostraram dor durante o período pós-operatório receberam $2 \mathrm{mg} / \mathrm{kg}$ de cloridrato de tramadol por via IM.

As variáveis paramétricas foram avaliadas pela ANOVA (Análise de Variância), seguida do teste de Tukey para comparação entre os diferentes tempos e grupos. As diferenças foram consideradas significativas quando $\mathrm{P}<0,05$. Os dados foram apresentados em tabelas e expressos como média \pm desvio padrão. Os testes estatísticos foram realizados em programa de computador (Instat Graphpad Software).

\section{RESULTADOS}

Foram observados valores significativamente menores na FC (M20 ao M40) no GI em relação à MPA e, em relação aos valores basais (M20), sem diferença significativa entre os grupos, com exceção do M30, em que a FC foi mais elevada no GII. A FR foi significativamente inferior aos valores basais do M20 ao M40 em ambos os grupos, sendo que no GII esta variável também foi inferior aos valores de MPA, nos momentos citados, e ao GI no M10 e M40. A temperatura retal foi inferior aos valores basais e à MPA (M20 ao M40) sem diferença entre os grupos.

No período pós-operatório, houve redução na FC em relação à MPA no $\mathrm{P} 30$ no GI; na FR, do P60 ao P120, no GI e do P30 ao P60 no GII em relação aos valores basais e, na temperatura reta, do P30 ao P90 em ambos os grupos e P120 no GII.

Após 15 minutos da administração da MPA, tanto no grupo I quanto no grupo II, os animais apresentaram-se atentos aos movimentos da sala e se mantendo em pé (escore 0); apenas um animal de cada grupo mostrou-se sonolento e sem coordenação dos membros (escore 1). Os animais de ambos os grupos apresentaram tônus do membro e mandíbula normais (escore 0) após 15 minutos da MPA, sendo que um animal do grupo I apresentou discreto relaxamento muscular (escore 1) e um do GII apresentou moderado relaxamento muscular (escore 2). Não foram observados efeitos adversos como excitação, tremores, vocalização, vômito ou defecação após a MPA.
No período pós-operatório, os animais se apresentaram sem dor e sem sinas de desconforto nos primeiros 30 minutos (escore-0), em ambos os grupos. No grupo I, um animal demonstrou dor leve sem sinais de desconforto (escore-1) aos 60 minutos e 3 animais apresentaram dor intensa com sinais óbvios de desconforto (escore-3) a partir dos 90 minutos. No grupo II, aos 120 minutos, um animal demonstrou sinal de dor moderada e algum sinal de desconforto (escore-2) e um animal apresentou dor intensa com sinais óbvios de desconforto (escore-3).

\section{DISCUSSÃO}

Os fármacos pertencentes ao grupo dos fenotiazínicos são utilizados primariamente para produzir sedação. Entretanto causam redução da pressão arterial dose-dependente devido ao bloqueio de receptores adrenérgicos periféricos do tipo $\alpha 1$, resultando em diminuição da resistência vascular periférica com consequente redução na pressão arterial [7,10]. A dose de $0,1 \mathrm{mg} / \mathrm{kg}$ de acepromazina, associada a opióides, pode reduzir a PAS em relação ao valor basal nos gatos, porém o mesmo não acontece com doses menores como a de $0,05 \mathrm{mg} / \mathrm{kg}$ [10]. Não foi observada diferença estatística quando a morfina $(0,5 \mathrm{mg} / \mathrm{kg})$ ou metadona $(0,5 \mathrm{mg} / \mathrm{kg})$ foram utilizadas associadas à acepromazina $(0,05 \mathrm{mg} / \mathrm{kg})$ pela via intravenosa em cães [13]. No presente estudo, apesar de se observar hipotensão já nos valores basais (valores inferiores a $100 \mathrm{mmHg}$ ), provavelmente pelo uso de manguito maior que $1 / 3$ do diâmetro do membro do animal, não foi identificada diferença entre os grupos e os tempos, apesar dos valores do período transoperatório serem levemente inferiores aos basais. O maior efeito hipotensor observado no transoperatório deve-se a potencialização produzida pelo propofol e halotano [15].

Os valores fisiológicos de frequência cardíaca em gatos variam entre 90 e 120 batimentos por minutos (bpm) em animais tranquilos, e entre 140 a $220 \mathrm{em}$ animais sob exame clínico [2]. Desta forma, os valores obtidos no exame pré-anestésico podem ser considerados fisiológicos, uma vez que se observou uma média de $174 \pm 36,16$ e $174,5 \pm 28,23$ batimentos por minuto, respectivamente, nos grupos I e II, já que estes animais poderiam estar apresentando ansiedade e desconforto durante a manipulação física.

Em geral, os opióides apresentam poucos efeitos cardiovasculares, e, por isso, são seguros para serem 


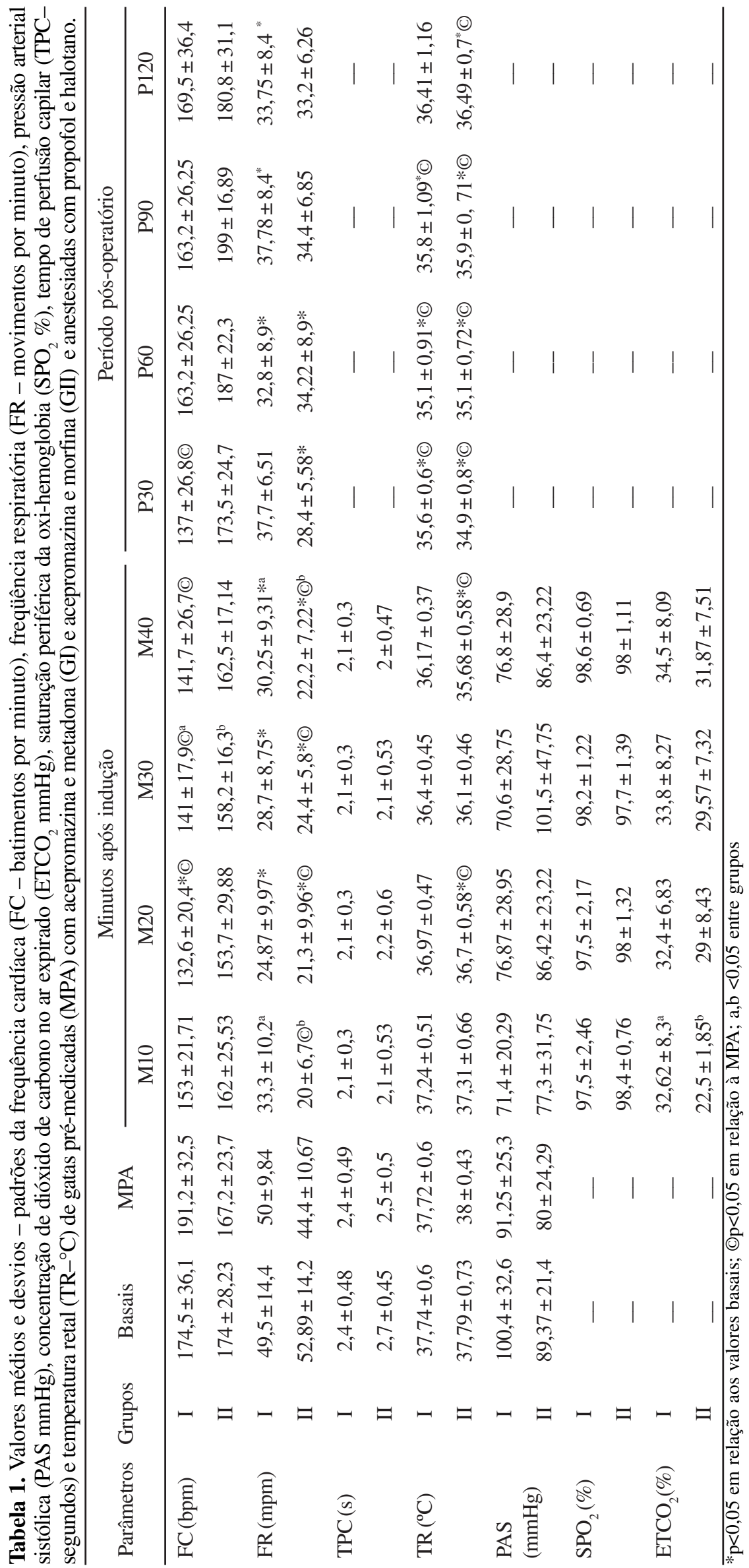


utilizados em animais doentes, debilitados ou cardiopatas [19]. Quando ocorrem, os efeitos mais comuns são a diminuição da frequência cardíaca e da pressão arterial [25]. Neste estudo, pode-se observar que não houve alteração na FC antes e após a MPA, em ambos os grupos, porém houve redução nesta variável no período transoperatório quando foi utilizado a associação de metadona, propofol e halotano, em relação aos valores obtidos 15 minutos após a MPA. Existem poucos estudos relatando os efeitos da metadona em felinos, no entanto a bradicardia não tem sido observada até então [14].

A depressão respiratória dose-dependente é um outro efeito colateral que pode ser atribuído ao uso dos opióides, especialmente os agonistas de receptores $m u$, como a morfina, metadona e fentanil, justificando a diminuição da FR significativa em relação aos valores basais observados durante o período transoperatório [2]. A morfina produz ação direta no centro respiratório, podendo proporcionar respiração regular e periódica, sendo que os efeitos da metadona são semelhantes [22]. Ainda, a associação com o propofol e o halotano pode ter potencializado a depressão do sistema respiratório neste estudo e em altas doses pode ocasionar parada respiratória [17].

Os centros de controle respiratório localizados no cérebro regulam a quantidade de dióxido de carbono que é expirado através do controle da velocidade e profundidade da respiração. Quando a respiração aumenta, a concentração sérica de $\mathrm{CO}_{2}$ diminui, levando à diminuição de $\mathrm{ETCO}_{2}$ [17]. Neste estudo, não foi possível obter os valores de $\mathrm{ETCO}_{2}$ basais e na MPA impossibilitando a determinação dos mesmos, sendo tal dado mensurado após a indução. Foi observado aumento na $\mathrm{ETCO}_{2}$ no final do período transoperatório em função da depressão respiratória ocasionada pelos fármacos, em ambos os grupos, embora os valores tenham se mantido próximo dos valores fisiológicos para a espécie [23]. Apesar da redução da frequência respiratória, em alguns momentos da avaliação, não foi observado grande incremento nos valores de dióxido de carbono expirado, demonstrando que a bradipneia não induziu hipoventilação significativa [5].

As causas básicas da hipotermia na cirurgia decorrem de inibição central termorregulatória e da elevada perda térmica que ocasionalmente ocorrem em associação prejudicando a termorregulação e redistribuição interna de calor, como resultado, verifica-se decréscimo na taxa metabólica. A associação de temperatura ambiente e efeito dos medicamentos pré-anestésicos e anestésicos levam a uma vasodilatação periférica, hiperventilação e a inexistência de atividade muscular agrava o quadro. Além disso, o sistema termorregulador hipotalâmico também é afetado pela administração de opióides [3]. A hipotermia é resposta comum, especialmente quando os opióides são utilizados durante a MPA, na presença de outras drogas depressoras do sistema nervoso central [8]. Esses fatores contribuíram para a hipotermia progressiva observada no presente estudo.

A identificação de dor em gatos é uma tarefa desafiadora, pois estes animais tendem a silenciar-se e ocultar seus sinais, em muitas ocasiões, eles tendem a esconder-se debaixo de cobertores ou até mesmo dentro de objetos, permanecendo imóvel ou rígido por longo período de tempo [12,20]. Neste trabalho, nos primeiros 30 minutos de pós-operatório, todos os animais se apresentaram sem sinais de dor, no entanto é difícil distinguir a analgesia da sedação em função dos efeitos sedativos residuais dos anestésicos, de modo que nas primeiras aferições pós-operatórias os animais podem não expressar sinais de dor [1]. Pode-se observar que poucos animais de ambos os grupos apresentaram sinais de dor no pós-operatório, sendo administrado cloridrato de tramadol $(2 \mathrm{mg} / \mathrm{kg} \mathrm{IM})$ para o alivio da dor. A ausência de diferença entre os grupos observadas neste estudo, em relação ao efeito analgésico, pode ser atribuída ao pequeno número de animais, ao modelo cirúrgico de dor (a ovariossalpingohisterectomia, apesar de excelente modelo para padronização da dor aguda pós-operatória em animais, pode não estimular desconforto suficiente para que a dor discreta a moderada seja avaliada em gatos) e ao sistema de escore empregado para aferição de dor [1].

Embora a associação de acepromazina e opióides tenha se mostrado uma técnica segura, capaz de provocar boa sedação no cão, isso não foi observado em gatos. Tem sido relatado na literatura que gatos tratados com doses elevadas de opióides podem apresentar excitação e, por essa razão, as doses utilizadas são mais baixas que as de cães. Como resultado, o grau de sedação e os efeitos colaterais produzido por essa associação é mínimo [7,21].

\section{CONCLUSÃO}

Pode-se concluir que ambas as associações produziram leve sedação e podem ser utilizadas com segurança em felinos com temperamento tranquilo, pois promoveram boa analgesia, sem efeitos colaterais. 


\section{NOTAS INFORMATIVAS}

${ }^{1}$ Acepran $1 \%$ - Univet. Cambuci, SP, Brasil.

${ }^{2}$ Mytedom - Cristália. Itabira, SP, Brasil.

${ }^{3}$ Dimorf - Cristália. Itabira, SP, Brasil.

${ }^{4}$ Propovan - Cristália. Itabira, SP, Brasil.

${ }^{5}$ Tanohalo - Cristália. Itabira, SP, Brasil.
${ }^{6}$ ECG PC VET - Tecnologia eletrônica brasileira. São Paulo, SP, Brasil.

${ }^{7}$ Doppller vascular portátil DV10 - Microem produtos médicos LTDA. Ribeirão Preto, SP, Brasil.

${ }^{8}$ Oximetro de Pulso Active X cap. - Ecafix- Industria e Comércio LTDA. São Paulo, SP, Brasil

\section{REFERÊNCIAS}

1 Bernardi C.A., Cassu R.N., Balan J.O., Fini O. \& Giuffrida R. 2008. Comparação do efeito analgésico da metadona via intramuscular ou epidural em gatas submetidas a ovariossalpingohisterectomia. 48f. Presidente Prudente, SP. Dissertação (Mestrado em Ciências Animal) - Programa de Pós-graduação em Ciência Animal, Universidade do Oeste Paulista.

2 Bonagura J.D. 1994. Cardiovascular diseases. In: Sherding R.G. (Ed.) The cat disease and clinical management. 2 nd edn. Philadelphia: Saunders, pp.819-946.

3 Alves M.B. 2007. Prevenção, controle e tratamento da hipotermia perioperatória em cães. 48f. Salvador, BA. Monografia (Curso de graduação em Medicina Veterinária), Universidade Federal da Bahia.

4 Fantoni D.T. \& Cortopassi S.R.G. 2002. Medicação pré-anestésica. In: Anestesia em cães e gatos. São Paulo: Roca, pp.151-158.

5 Fantoni D.T. \& Mastrocinque S. 2002. Fisiopatologia e controle da dor. In: Cotorpassi S.R.G \& Fantoni D.T. (Eds). Anestesia em cães e gatos. São Paulo: Roca, pp.324-334.

6 Gorniack S.L. 1996. Hipnoanalgésicos e neuroleptoanalgesia. In: Spinosa V., Gorniack S.L. \& Bernardi M.M. (Eds). Farmacologia aplicada à medicina veterinária. 4 ed. Rio de Janeiro: Guanabara Koogan, pp.142-143.

7 Hall L.W., Clarcke K.W. \& Trim C.M. 2001. Principles of sedation, analgesia and premedication. In: Veterinary anaesthesia. 10th edn. London: WB Saunders, pp.75-112.

8 Lamont L.A. \& Mathews K.A. 2007. Opioids, Nonsteroidal Anti-inflamatories, and Analgesic Adjuvantes In: Tranquile W.J., Thurmon J.C. \& Grimm K.A. (Eds). Lumb \& Jone's, Veterinary Anesthesia and Analgesia. 4th edn. Sydney: Blackwell Publishing, pp.241-271.

9 Lascelles B.D. \& Waterman A.E. 1997. Analgesia in cats. Veterinary clinics of North America-Small Animal Pratice. 4: 203-213.

10 Ludders J.W. 1983. Blood pressure response to phenylephrine infusion in halothane-anesthetized dogs given acetylpromazine maleate. American Journal of Veterinary Research. 44: 996-999.

11 Clinica Perionatal. 2002. Analgesia opióide em recém-nascidos na unidade de terapia intensiva, 493-509p. Disponível em: <http://www.paulomargotto.com.br/documentos/analgesia.doc> acessado em 02/2009

12 Mathews K.A. 2000. Pain assessment and general approach to management. Veterinary clinics of North America-Small Animal Pratice. 30: 729-755.

13 Monteiro E.R., Rodrigues Junior A., Assis H.M.Q., Campagnol D. \& Quitzan J.G. 2009. Comparative study on the sedative effects of morphine, methadone, butorphanol or tramadol, in combination with acepromazine, in dogs. Veterinary Anaesthesia and Analgesia. 36: 25-33

14 Monteiro E.R., Picoli F.M., Queiroz M.Q.O., Campagnol D. \& Quitzan J.G. 2008. Efeitos sedativo e cardiorrespiratório da administração da metadona, isoladamente ou em associações à acepromazina ou xilazina, em gatos. Brazilian Journal of Veterinary Research and Animal Science. 45: 289-297.

15 Muir III W. W., Hubbell J.A., Skarda R. T. \& Bednarski R.M. 2001. Fármacos específicos para anestesia intravenosa. In: Manual de Anestesia Veterinária. 3.ed. Porto Alegre: Artmed, pp.122-123

16 Miyake R.S., Reis A., Grisi S. 1998. Sedação e analgesia em crianças. São Paulo: Revista de Associação Médica Brasileira. 1: $56-64$

17 Paddleford R.R., Hartsfield S.M., Haskins S.C., Sedgwick C.J. \& Trim C.M. 2001. Drogas pré-anestésicas. In: Manual de anestesia em pequenos animais. 2.ed. São Paulo: Roca, pp.15-35

18 Parramon F., Garcia C., Gambus P., Vilaplana J., Aragones N. \& Villalonga A. 2003. La analgesia postoperatoria controlada por el paciente es más efectiva con metadona epidural que con metadona endovenosa en la cirugía torácica. Revista Española de Anestesiología y Reanimación. 50: 326-331

19 Sackman J.E. 1997. Pain and it's management. Veterinary Clinics of North America: Small Animal Practice. 6: 1487-1503

20 Smith J.D., Allen S.W., Quandt J.E. \& Tackett R.L. 1996. Indicators of postoperative pain in cats an correlation with clinical criteria. American Journal of Veterinary Research. 57:1674-1678 
Manfrinate R., Dahroug M.A.A., Farias D.C., Vasconcelos L.P.S., Wayhs N.N.S., Monzem S., Silva E.C., Galceran J.V.A., Bogorni F.,

Souza R.L. \& Guimarães L.D. 2009. Efeitos da morfina e da metadona associadas... Acta Scientiae Veterinariae. 37(3): $245-251$.

21 Stepien R.L. 1995. Cardiorespiratory effects of acepromazine maleate and buprenorphine hydrochloride in clinically normal dogs. American Journal of Veterinary Research. 56:78-84.

22 Short C.E. 1987. Pain, analgesics, and related medications. In: Principles e practice of veterinary anesthesia. Baltimore: Williams \& Wilkins, pp.34-37.

23 Thurmon J.C., Tranquilli W.J. \& Benson. G.J. 1996. Preanesthetics and anesthetic adjuncts In: Lumb \& Jones'veterinary anesthesia. 3rd edn. Baltimore: William \& Wilkins, pp.183-209.

24 Wagner A.E. 2002. Opoids. In: Gaynor J.S. \& Muir W.W. (Eds). Veterinary pain management. St. Louis: Mosby, pp.164-183.

25 Werner B.E. \& Taboada J. 1994. Use of analgesics in feline medicine. Compendium on Continuing Education for the Practicing Veterinary. 4: 493-499. 